\title{
Wide Area Observation of Fully Hydrophilic Tissue Achieved by Sliding It on the Dish of the Atmospheric Scanning Electron Microscope (ASEM)
}

\author{
Chikara Sato ${ }^{1,2}$ Mari Sato $^{1}$, Tatsuhiko Ebihara ${ }^{1}$, Hiditoshi Nishiyama ${ }^{3}$, Mitsuo Suga ${ }^{3}$, Nassirhadjy \\ Memtily ${ }^{1,2,4}$
}

1. Biomedical Research Institute, National Institute of Advanced Industrial Science and Technology (AIST), Higashi 1-1-1, Tsukuba, Ibaraki 305-8566, Japan.

2. Graduate School of Comprehensive Human Sciences, University of Tsukuba. 1-1-1 Tennodai, Tsukuba, Ibaraki Prefecture 305-0006, Japan.

3. Advanced Technology Division, JEOL Ltd., Musashino 3-1-2, Akishima, Tokyo 196-8558, Japan.

4. Traditional Uyghur Medicine Institute of Xinjiang Medical University, 393 Xinyi Rd, Urumqi, Xinjiang Uyghur Autonomous Region, 830011 China.

Correspondence should be addressed to Chikara Sato (ti-sato@aist.go.jp).

The ASEM has an inverted SEM configuration, and was developed to realize SEM observation of a sample in aqueous liquid in a readily-accessible, open container (ASEM dish) [1]. An optical microscope $(\mathrm{OM})$ positioned above the dish can be used to observe wide areas of the sample, and smaller regions can be observed by the SEM through a thin silicon nitride (SiN) film in the base of the ASEM dish (Fig. 1A). The optical axes of both microscopes are aligned and fixed to ensure that correlative images are recorded, and the specimen stage can move two-dimensionally (x-y) for targeting. From below the ASEM dish, the inverted SEM projects an electron beam up through the SiN film to the sample. Backscattered electrons are captured for ASEM imaging by a disk-shaped detector located just beneath the $\mathrm{SiN}$ film. The specimen depth observable by ASEM is $2-3 \mu \mathrm{m}$ at $30 \mathrm{kV}$, and the resolution is $8 \mathrm{~nm}$ near the SiN membrane [1-2]. ASEM was widely applied to observe various primary cells, tissues, bacteria and protein crystals [3-7]. The OM makes the handling of specimens easy, which is exploited here for the wide area observation necessary for intra-operative cancer diagnosis.

For wide area observation using ASEM, a fixed tissue slab in a radical scavenger (glucose) solution is repeatedly pushed slightly to the side with tweezers under monitoring by OM from above, and imaged by SEM from below (Fig. 1A) [8]. This can be done using a standard ASEM dish with a single SiN window (Fig. 1B) or a newly developed 8-window ASEM dish (Fig. 1C). In the example shown, a slab of spinal cord tissue stained with PTA was placed in an 8-windowed ASEM dish, and repeatedly induced to slide very slightly across the thin membrane windows and imaged. Although the observable area in each ASEM imaging was restricted to the field of the 8 windows, two sequential images partly overlapped and could be merged, covering a wider area of the spinal cord (Fig. 1D, E). The observable area of the multi-windowed dish was thus successfully extended. The shift of the tissue on the flat bottom surface of ASEM dish (Fig. 1A) was precisely monitored by OM from above. ASEM realize high throughput observation of wet tissues, and could improve intraoperative cancer diagnosis because ASEM does not require the cryo-thin-sectioned OM which takes about 15-30 minutes for each sample.

Skeletal muscle fibers were clearly visible when PTA-stained tissue slabs of gastrocnemius muscle were observed by ASEM (Fig. 2). In myocytes, I-bands and A-bands were visualized as dark zones and broader bright zones, respectively, and Z-lines were distinguished as a fine thin line in the center of the dark I-bands (Fig. 2B, C). These results suggest that protein complexes in natural aqueous liquid environment can be studied at high resolution in combination with tissue engineering using the ASEM. 


\section{References}

[1] H Nishiyama et al, J Struct Biol. 172 (2010), p. 191-202.

[2] Y Maruyama et al, J Struct Biol. 180 (2012), p. 259-270.

[3] C Sato, JEOL news 46(1) (2011), p.17-22.

[4] K Hirano et al, Ultramicroscopy143 (2014), p. 52-66.

[5] T Kinoshita et al, Microsc Microanal 20(2) (2014), 469-483.

[6] C Sato et al, Biochem Biophys Res Commun 417(2012), p1213-1218.

[7] Y Maruyama et al, Int J Mol Sci 13(8) (2012), p.10553-10567.

[8] N Memtily et al, International J Oncology, in press.
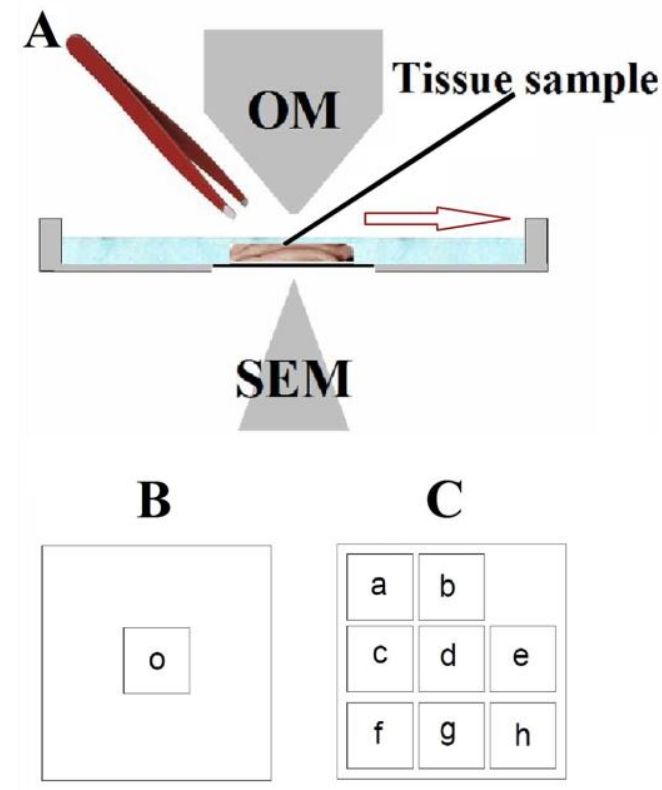

D

$\mathbf{E}$
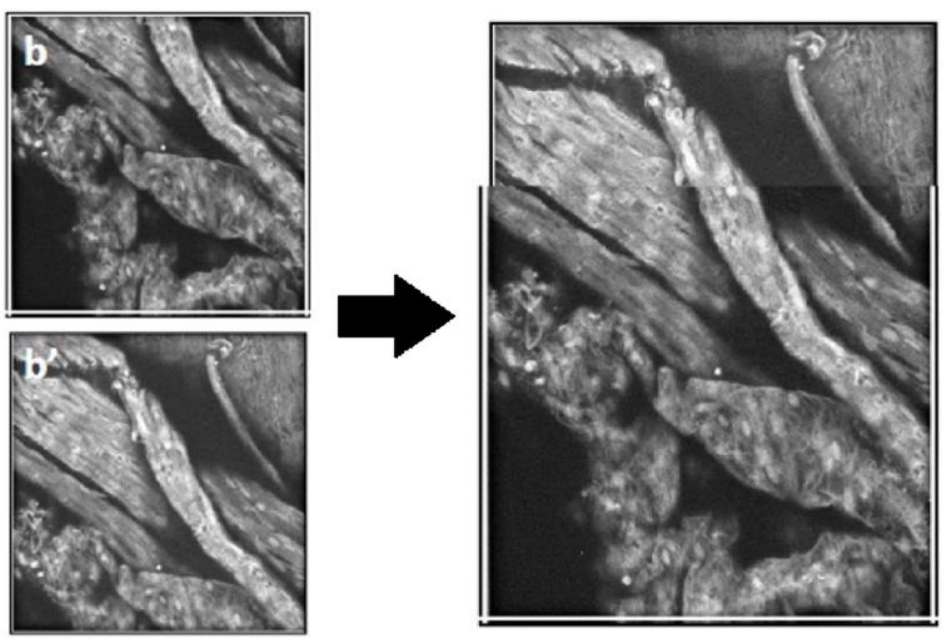

Figure 1. Wide area imaging by shifting a tissue on the ASEM dish. (A) Schematic diagram of the procedure. The shift caused by pushing with the tweezers is precisely controlled by low magnification monitoring using the upper OM. (B) Schematic diagrams of a one-window and an 8-window ASEM dish (C). All windows are $250 \times 250 \mu \mathrm{m}$. (D) Spinal cord image initially recorded from window b and the image recorded in the same window after the tissue has been pushed in one direction causing it to slip across the SiN-film window, b'. (E) Merged image of windows b and b'.
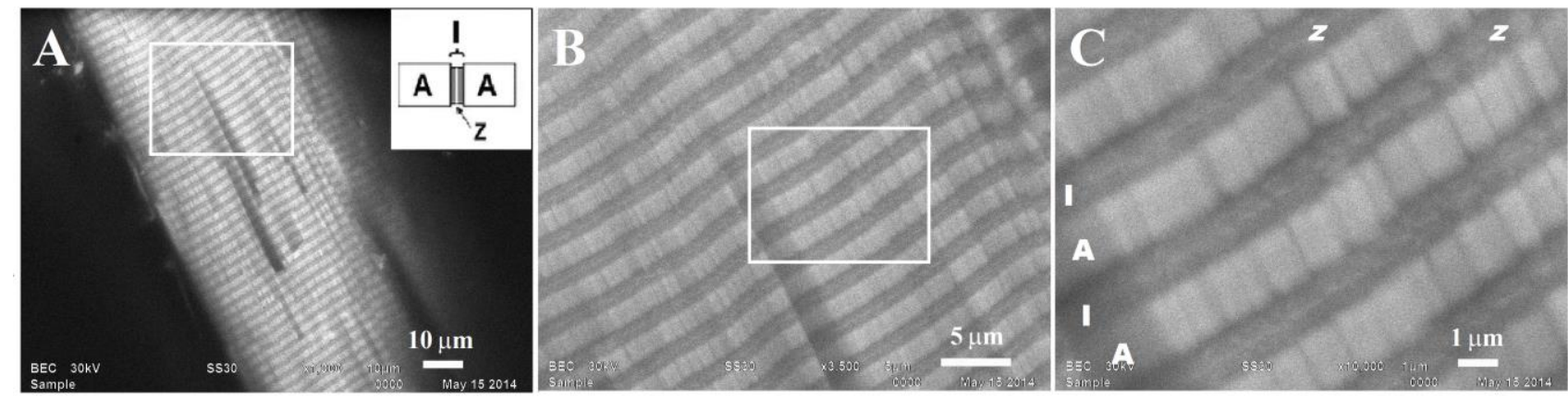

Figure 2. ASEM images of gastrocnemius muscle stained with PTA. (A) Low magnification image. Filaments run along striated muscle fibers. (B, C) Higher magnification image of the white rectangle in the preceding panel. The muscle fiber has A-bands, evident as broad bright zones (A), and I-bands, evident as dark zones (I). Z-lines, look like a faint thin white line in the middle of the I-bands (I). Scale bar $10 \mu \mathrm{m}$ in $\mathrm{A}, 5 \mu \mathrm{m}$ in B and $1 \mu \mathrm{m}$ in $\mathrm{C}$. 